\title{
An approach of zirconium oxide/polyethylene glycol nanocomposite film on screen printed carbon electrode and its application in glucose determination
}

\begin{abstract}
An amperometric biosensor for the quantitative measurement of glucose is reported. The biosensor is based on a biocomposite that is homogeneous and easily prepared. This biocomposite is made of polyethylene glycol (PEG), Cetyl trimethylammonium bromide (CTAB), glucose oxidase (GOx), and zirconium oxide ( $\mathrm{ZrO} 2)$ nanoparticles. Effect of applied potential, $\mathrm{pH}$, nanocomposite layer, ratio of zirconium oxide nanoparticles ( $\mathrm{ZrO} 2)$ to polyethylene glycol (PEG), concentration of ferrocenecarboxaldehyde $(\mathrm{Fc})$ and concentration of enzyme that governed the analytical performance of the biosensor have been studied. The biosensor was applied to detect glucose with a linear calibration range from $0.1 \mathrm{mM}$ to 12 $\mathrm{mM}$ and limit of detection $0.04 \mathrm{mM}$. The variation coefficient (RSD) for repeatability was $7.5 \%$ for ten successive assays while $6.01 \%(n=5)$ for reproducibility test demonstrating that the biosensor fabrication process is reproducible. Common interfering compounds on the amperometric response of the sensor were investigated and discussed herein.
\end{abstract}

Keyword: Biosensor; Polyethylene glycol; Zirconium oxide 